\title{
ASSOCIATION BETWEEN SOCIAL SUPPORT AND QUALITY OF LIFE IN PATIENTS WITH BREAST CANCER AT HIWA CANCER HOSPITAL IN SULAIMANI CITY/IRAQ
}

\author{
Avin Anwar Mahmood ${ }^{1}$, Muhammad Rashid Amen 2 * \\ 1. University Nurse at Hiwa Cancer Hospital / General Directorate of Health in Sulaimani Governorate; \\ 2. Department of Ault Nursing, College of Nursing, University of Sulaimani, City of Sulaimani, Iraq.
}

\section{Corresponding author: Muhammad Rashid Amen}

Email: muhammad.amen@univsul.edu.iq

\section{ORCID}

\section{ABSTRACT}

Background: Breast cancer is the most common cancer in women worldwide. During and after treatments, breast cancer patients may vulnerable for problems and in need of support. Receiving social support may reduce patients' stress, help to cope with the situation, and improve patients' quality of life.

Aim: The aim of the study was to assess perceived social support, quality of life, and explore the association between them in a sample of females' breast cancer.

Methodology: Three hundred Forty-Two patients with breast cancer who visited the outpatient clinic at Hiwa Hospital in Sulaimani City were recruited to the current cross-sectional study. The data was collected at least 3 months postchemotherapy, the direct interview was used to fill a questionnaire that includes participants' characteristics, source of social support, types of treatment, Multidimensional Scale of Perceived Social Support to assess perceived social support, and European Organization for Research and Treatment of Cancer (EORTC QLQ C-30, EORTC QLQ BR-23) to assess patients' quality of life. Statistic Package of Social Science 24 software was used for statistical analysis.

Results: Participants' mean age was $(42.65 \pm 7.71)$, total social support score was $(52.68 \pm 20.09)$ on a scale of (12 to 84), about half (47.4\%) received it moderately. The family was the highest source of social support (19.89 \pm 7.80$)$. Global health state score was (68.52 \pm 19.80$)$, emotional was the most affected functional subscale (52.10 \pm 29.60$)$. Regarding the symptom's subscale, the financial deficit $(55.95 \pm 33.07)$ was the most disrupted symptom followed by fatigue (43.60 \pm 21.16$)$. Furthermore, sex function (32.99 \pm 28.79$)$ was a more impaired aspect among breast cancerspecific functions, and hair loss (59.84 \pm 41.37$)$ was the most symptom reported by the patients. Moreover, perceived social support is associated and correlated with participants' quality of life, $(p<0.05)$.

Conclusion: Kurdish breast cancer patients perceived moderate social support, the global health status was moderate to good, most affected function was emotional and sex functions, financial deficit, fatigue, and hair loss were most disrupting symptoms. Social support enhanced patients' QoL in term of enhancing functions and minimized symptoms.

Keywords: Breast Cancer, Quality of Life, Social Support

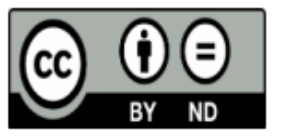

This work is licensed under a Creative Commons Attribution Non-Commercial 4.0 International License.

Received: 29 July 2021, Accepted: 27 November 2021, Available online: 20 January 2022 


\section{INTRODUCTION}

Breast cancer is the most commonly diagnosed malignancy and the leading cause of death among women worldwide. It is revealed that breast cancer alone is responsible for $30 \%$ of newly diagnosed cancer cases in women and there is a probability that one in eight women will develop breast cancer in her lifetime, while $14 \%$ of cancerrelated deaths are attributed to it (Siegel et al, 2017). Breast cancer is one of the most common and fatal diseases in the female population, which has a great impact on the psychological, emotional, social and family life of breast cancer patients. In the Kurdistan region, the incidence of breast cancer is growing, it showed an escalating trend in last decade, 94 new breast cancers registered at Hiwa Hospital in 2006, while it increased to 531 in 2019 (Hiwa Hospital report, 2020).

Being diagnosed with breast cancer is a very stressful event and has tremendous consequences for most persons who experience it, affecting all aspects of life and the temporary side effects associated with the treatment may influence the patients' health related quality of life during and post treatment (Hassen et al 2019). The increase of morbidity in breast cancer afflicted patients, and aggravation for disease burden put a longterm impact on the physical and psychological health of patients, malignancies, especially in advanced stages, are associated with a compromised quality of life (QoL), which can be attributed to physical, psychological and social factors. While comparing cancer survivors with the control population, it is observed that they demonstrate a higher fatigue score and poorer quality of life (Wang et al, 2020). Simultaneously, physical impairment and psychological distress or emotional changes will have a negative influence on quality of life (QOL), its impact is not only confined to the treatment phase but also in the post treatment period (Oh and Cho 2020).

Social support is the interrelationships that protects individual from stress, reduces an illness distress and creating a sense of psychological and physical well-being and has a profound impact on quality of life in patients with chronic diseases, it has been found to be correlated with positive treatment outcomes for many chronic conditions including breast cancer, and it significantly reduces the stress emanating from cancer diagnosis as well as improves emotional wellbeing (Kim et al 2010) (Adam \& Koranteng 2020). Social support is usually provided by family members, relatives, friends and other relevant persons or facilities (Alshraifeen et al, 2020). Breast cancer and its treatment causes considerable harm to women in various life aspects including wellbeing, physical, psychological and social problems. Among breast cancer patients, inadequate social support is associated with a substantial increase in cancer-related mortality, increased social support, on the other hand, shows positive effects on breast cancer patients' physical, psychological and social functioning and on their QoL. (Salakari et al, 2017)

Furthermore, Celik et al. (2021) found that social support might transform patients' fear of progression and help them cope with uncertainty, in which way the patients had a better performance in adapting to society and in turn improved their QOL. Ban et al (2021) assessed the effect of social support by identifying the linkage between fear of progression and QOL for breast cancer patients, the result indicated that social support as an important factor in improving QOL.

As described previously, assessing the quality of life and perceived social support may have numerous benefits including the ability to provide clinicians and patients with accurate expectations about the likely impact of treatments on wellbeing and functioning, the ability to identify common problems that will need to be addressed, and the ability to identify therapies and interventions effective in addressing these problems. In addition, determine the association between QoL and social support may improve clinicians' ability to predict treatment response and survival time in certain contexts. Besides, numerous studies have found that higher social support led to a better quality of life which may associate with longer survival of patients with breast cancer. Therefore, the aim of the present study was to assess QoL and perceived social support and find out the association between them in the women with breast cancer.

\section{METHOD}

Out of 413 breast cancer patients visited outpatients' clinics at Hiwa Hospital, 342 patients were recruited into the study who met eligibility criteria, non-probability convenience sample technique was used. We included patients who were willing to participate, currently following up for any form of treatment and their last chemotherapy session was before at least 3 months. We excluded those patients who were diagnosed with any mood disorder before cancer, an uncontrolled chronic medical illness which not related to cancer or its treatment-related complications, and severe physical disability not related to cancer or its treatment.

The data for present cross-sectional study collected through constructed questionnaire which includes participants sociodemographic and clinical characteristics, Multidimensional Scale of Perceived Social Support (MSPSS), European Organization for Research and Treatment of 
Cancer Quality of Life questionnaire core 30 (EORTC QLQ C-30) and (EORTC QLQ BR-23).

The researcher collected data through direct face-to-face interview, a separate comfortable room was used for this reason, a brief explanation of the aim of the study was carried out prior to data collection. Moreover, pre-test was done on 25 patients to identify clarity and applicability of the tools, and to provide feedback about the questionnaire.

\section{-Study instruments}

Social support

Social support was measured by the Multidimensional Scale of Perceived Social Support (MSPSS). This 12 -item measure is scored on a 7-point, Likert-type scale that ranges between $1=$ very strongly disagree and $7=$ very strongly agree (Canty-Mitchell \& Zimet, 2000) and assessed the three sources of family, friends, and significant others support.

MSPSS sub-scales are composed of four items. Overall social support scores are calculated by summing the scores for each item, with higher scores indicating higher levels of social support. Subjects can be categorized according to the level of social support they receive as having low, medium, and high if they achieve total scores of (1-2.9), (3-5), and (5.1-7), respectively or for the total of MSPSS other format can be use (12-35) low, (36-60) medium, (61-84) high perceived social support (Zimet et al 1988). The reliability and validity of the MSPSS have been demonstrated across several different samples (Canty-Mitchell \& Zimet, 2000). In the present study Cronbach's alpha coefficient of 0.90 was reported for the total scores and $0.92,0.90$, and 0.90 for the family, friends, and significant others subscales, respectively. At the time of development, the Cronbach's a reliability was .91; Cronbach's a for each subscale ranged from 0.90-0.95. In this study, the Cronbach's a of the social support scale was 0.95 .

\section{Quality of Life}

The validated European organization for research and treatment of cancer quality of life questionnaire core 30 (EORTC QLQ C-30) was used to measure breast cancer patients' health-related quality of life in addition to European organization for research and treatment of cancer quality of life questionnaire specific for breast cancer (EORTC QLQ BR23) was used to assess specific factors of breast cancer patients' QOL. Functional and symptom items of the EORTC QLQ-C30 and QLQ-BR23 questionnaires were rated on a 4-level response system from (1) not at all to (4) very much, while the Global health status/QOL (Question29, Question30) used a 7-point response scale.

The standardized tool EORTC QLQ-C30 was developed through a process of international joint study from multiple countries, and it is the most widely used standardized tool to measure the quality of life of cancer patients. This tool is composed of three subdomains and 30 items. It includes two items on Global health status/quality of life and five functional domains; physical, role, cognitive, emotional, and social functioning that include 15 items; three symptom domains; fatigue, pain, nausea/vomiting that include seven items; and one item for each of the symptoms commonly reported by cancer patients; dyspnea, loss of appetite, insomnia, constipation, diarrhea, and financial deficit. The EORTC QLQ-C3O is converted into a score ranging between $(0-100)$ points; higher global health/quality of life scores, higher functional domain scores, and lower symptom domain scores indicate higher quality of life. Moreover, overall quality of life can be understood as a measurement of comprehensive quality of life (Fayers et al 2001).

Likewise, the QLQ- BR23, which assesses the QOL of breast cancer patients, has 23 items assessing disease symptoms and functions. The breast cancer module is designed for patients with different disease stages and treatment modalities. The module comprises 23 items, which incorporate 5 multi-item scales to assess systemic therapy side effects, arm symptoms, breast symptoms, body image, and sexual functioning. In addition, single items assess sexual enjoyment, hair loss, and future perspective (Sprangers et al, 1996).

\section{Statistical analysis}

Data analysis was performed using Statistical Package for Social Science (SPSS)-Version 24. All categorical data were presented in frequency and percentage and continuous data were presented in mean and standard deviation. Descriptive statistics were used to describe the participants' demographics and cancer-related information. Pearson's correlation coefficients were used to examine the correlation between social support and QOL scores. Chi-square was used to determine the association between the level of perceived social support and the QOL subscale. The statistical significance level was set at $p \leq 0.05$.

\section{RESULTS}

In total, 342 women were willing to participate in the study. The mean age was $(42.65 \pm 7.71)$ years, ranged from 25 to 79 years, almost (63\%) less than 
45 years. The distribution of marital status was almost as follows: married or living with a husband (79\%), widowed/divorced (6\%), and single $(15 \%)$. Concerning education, more than (20.5\%) with no formal education, (36.3\%) completed primary school, and (33.3\%) university graduated. Regarding participants' occupations, the majority almost $(82 \%)$ were housewives. The income for more than $(60 \%)$ of women was less than their expenditure and for (38\%) was equal, Table (1).

The proportion of the obese and overweight as illustrated in table 2 was (57.3\%) and (35.7\%) respectively, a few $(7 \%)$ were normal. The affected breast for more than half $(51.5 \%)$ was the right side. The average age of diagnosis was $(39.92 \pm 7.27)$ years, almost $(10 \%)$ were diagnoses with breast cancer earlier than the age of 30 years, the proportion of patients diagnosed at (30 - 39) and (40 - 49years was (45.6\%) and (38\%) respectively, while the percentage of the participant diagnosed at 50 years and older was $(6.4 \%)$ only.

The frequency of breast cancer treatments was; mastectomy (52.6\%), lumpectomy (47.4\%), radiotherapy $(85.4 \%)$, biological $(21.1 \%)$, and rehabilitation $(27.5 \%)$ as shown in table (3).

Patients with breast cancer had a total MSPSS score of $(12-84)$, with a mean score of $(52.68 \pm$ 20.09) (Table 4). It was found that $(48.0 \%)$ of the patients had high social support, (31.5\%) had medium social support, and $(20.5 \%)$ had low social support (Table 5). The best mean source of social support on the MSPSS subscale was found to be family $(4.97 \pm 1.96)$ and significant other $(4.74 \pm$ $1.86)$, however, the worst score was to the friend $(3.47 \pm 102.8)$ on the scale of $(1-7)$ (Table). The proportion of high social support received from family was highest $(52.8 \%)$, when compared to significant other (48.2\%) and friends (34.5\%).

The result of present study reveals that the family was greater social support source, more than half $(52.1 \%)$ received high social support from family, followed by significant others (48\%) for participant reported that had high social support from physicians, nurses and other health care professionals. The proportion of high social support provided by friend was (34.5\%) only. Regarding total social support (31\%) perceived high social support, the proportion of medium social support was $(47.4 \%)$ and $(21.6 \%)$ of them perceived low social support level, as shown in Table (5).

Overall, for EORTC-C30 table (6), the mean of global health status was $(68.52 \pm 19.80)$; the functional scale was low especially in emotional functioning and cognitive functioning (52.10 \pm
$26.60)$ and $(54.97 \pm 26.99)$ respectively. The role functioning had the highest score $(68.42 \pm 25.87)$ followed by social functioning and physical functioning $(67.54 \pm 28.31)$ and $(66.12 \pm 20.11)$ respectively and the total functional scale score was $(61.83 \pm 17.61)$. While symptom scales were moderate-to-low for most items, the higher score was for financial deficit $(55.95 \pm 33.07)$ and the lowest score was $(3.70 \pm 11.11)$ diarrhea, other symptoms which have high scores were fatigue, pain, and insomnia (43.60 \pm 19.80$)$, (39.96 \pm $24.15)$, and (29.24 \pm 27.82$)$ respectively, and total symptoms scores were $(25.37 \pm 14.08)$.

The scores of QoL according to QLQ-BR23, patients exhibited higher scores for future perspective and body image $(5.83 \pm 19.97)$ and $(52.87 \pm 29.44)$ respectively, while the least score was for sexual functioning $(32.99 \pm 28.79)$ and total functional scale score was $(45.81 \pm 17.83)$. Regarding symptom scales, hair loss, arm symptoms, and systemic therapy's side effects were more disturbing $(59.84 \pm 18.53),(43.27 \pm$ $20.05)$, and $(41.35 \pm 18.53)$ respectively, followed by breast symptoms $(32.36 \pm 22.27)$ and the score of total symptoms subscale was $(44.21 \pm 19.76)$ as presented in Table (7). The higher scores in global health status showed better QoL. Similarly, higher scores in functional scales indicate better QoL. On the other hand, higher scores in symptom scales show worse QoL.

The correlations between the quality of life measured using EROTC QOL-C30 EROTC QOL-BR23 and social support are presented in Table 8. A most strong positive correlation was found between MSPSS and each of QOL-C30 and QOLBR23 functional subscales, ( $r: 548 ; p: 0.001)$ and ( $r: 502 ; \mathrm{p}: 0.001)$ respectively. In addition, a negative correlation was found between MSPSS and each of QOL-C30 and QOL-BR23 symptoms subscales ( $r:$ - 454; p:0.001) and ( $r:-372 ; p: 0.001)$ respectively. Moreover, MSPSS correlated significantly to global health status/QOL ( $r: 436$; $\mathrm{p}: 0.001)$. All reported significant correlations to show a positive relationship between the quality of life and social support, which mean the more social support an individual receives, the higher his or her quality of life is. Table (8).

As demonstrated in tables $(9 \& 10)$ the scores of global health status/QoL (77.83 \pm 15.32$)$ and QOLC30 functions (71.31 \pm 10.42$)$ were significantly higher among patients received high social support when compared to those who received low social support $(57.66 \pm 24.95)$ and $(48.85 \pm$ $15.08)$ respectively. Whereas, it was the opposite regarding symptom subscales scores in both QoL scales. This reflects better QoL among patients who perceived higher social support. Social function in the QOL-C30 and future perspective in 
the QOL-BR23 had greater scores $(83.02 \pm 17.75)$ and $(67.93 \pm 25.29)$ respectively among patients who perceived high social support when compared to other functions.

A significant association was found between social support with all functions in both QOL- C30 and QOL-BR23 except sexual enjoyment. In addition, high social support is associated with lower symptoms of most QOL-30 and QOL-BR23 symptom subscales. Fatigue, pain, dyspnea, insomnia, appetite loss, and financial deficit scores on the QOL-C30 scale were decreased with increasing social support, on another hand systematic treatment adverse effect, arm, and breast symptoms decreased among high perceived social support patients, $(p<0.05)$.

\begin{tabular}{|c|c|c|}
\hline Characteristics & Frequency (342) & $\%$ \\
\hline \multicolumn{3}{|l|}{ Age Groups } \\
\hline Up to 45 Years & 216 & 63.2 \\
\hline$\geq 45$ Years & 126 & 36.2 \\
\hline Mean \pm SD & \multicolumn{2}{|c|}{$42.65 \pm 7.71$} \\
\hline \multicolumn{3}{|l|}{ Marital Status } \\
\hline Married & 270 & 78.9 \\
\hline Single & 52 & 15.2 \\
\hline Widows/Separate & 20 & 5.8 \\
\hline \multicolumn{3}{|l|}{ Levels of Education } \\
\hline Illiterate / No formal education & 70 & 20.5 \\
\hline Primary & 124 & 36.3 \\
\hline Secondary & 24 & 9.9 \\
\hline Institute/University & 114 & 33.3 \\
\hline \multicolumn{3}{|l|}{ Occupations } \\
\hline Housewives & 280 & 81.9 \\
\hline Paid Employed & 62 & 18.1 \\
\hline \multicolumn{3}{|l|}{ Financial Status } \\
\hline Income < Expenditure & 206 & 60.2 \\
\hline Income = Expenditure & 130 & 38.0 \\
\hline Income > Expenditure & 6 & 1.8 \\
\hline Total & 342 & 100 \\
\hline
\end{tabular}

Table (2) Distribution of sample according clinical characteristics

\begin{tabular}{|c|c|c|}
\hline Characteristics & Frequency & $\%$ \\
\hline \multicolumn{3}{|l|}{ Body Mass Index } \\
\hline Normal & 24 & 7.0 \\
\hline Over-Wight & 122 & 35.7 \\
\hline Obese & 196 & 57.3 \\
\hline \multicolumn{3}{|l|}{ Affected Breast } \\
\hline Left & 144 & 42.1 \\
\hline Right & 176 & 51.5 \\
\hline Left \& Right & 22 & 6.4 \\
\hline \multicolumn{3}{|l|}{ Age at Diagnosis } \\
\hline$<30$ Years & 34 & 9.9 \\
\hline $30-39$ Years & 156 & 45.6 \\
\hline 40 - 49 Years & 130 & 38.0 \\
\hline$\geq 50$ Years & 22 & 6.4 \\
\hline Mean SD & \multicolumn{2}{|c|}{$39.92 \pm 7.27$} \\
\hline \multicolumn{3}{|l|}{ Stage of Cancer } \\
\hline $1^{\text {st. }}$. & 40 & 11.7 \\
\hline $2^{\text {nd }}$ & 170 & 49.7 \\
\hline $3^{\text {rd }}$ & 128 & 37.4 \\
\hline $4^{\text {th }}$ & 4 & 1.2 \\
\hline Total & 342 & 100 \\
\hline
\end{tabular}

Table (3) Distribution of sample according type of treatments

\begin{tabular}{lcc}
\hline Type of Treatments & Frequency & $\%$ \\
\hline Types of Surgery & & \\
Lumpectomy & 162 & 47.4 \\
Mastectomy & 180 & 52.6 \\
Radiotherapy & & 85.4 \\
Yes & 292 & 14.6 \\
No & 50 & 21.1 \\
Biological Therapy & & 78.9 \\
Yes & 72 & \\
No & 270 & 27.5 \\
Rehabilitation & & 72.5 \\
Yes & 94 & 100 \\
\hline No & 248 & \\
\hline
\end{tabular}


Table (4) Distribution of MSPSS and its subscale scores

\begin{tabular}{lcccc}
\hline MSPSS Subscales & Mean & SD & Minimum & Maximum \\
\hline Significant Other Support & 4.74 & 1.86 & 1 & $\mathbf{7}$ \\
Family Support & 4.97 & 1.96 & 1 & $\mathbf{7}$ \\
Friend Support & 3.47 & 2.10 & 1 & $\mathbf{7}$ \\
Total MSPSS & 52.68 & 20.09 & 12 & $\mathbf{8 4}$ \\
\hline
\end{tabular}

MSPSS: Multidimensional Scale of Perceived Social Support, SD: standard deviation

Table (5) Distribution of levels of total social support and subscales.

\begin{tabular}{lcc}
\hline MSPSS Subscales & Frequency & $\%$ \\
\hline Significant Other Social Support & 70 & 20.5 \\
Low Social Support & 108 & 31.5 \\
Medium Social Support & 164 & 48.0 \\
High Social Support & & 18.1 \\
Family Social Support & 62 & 29.8 \\
Low Social Support & 102 & 52.1 \\
Medium Social Support & 178 & 45.6 \\
High Social Support & & 19.9 \\
Friend Social Support & 156 & 34.5 \\
Low Social Support & 68 & \\
Medium Social Support & 118 & 21.6 \\
High Social Support & & 47.4 \\
Total MSPSS social Support & 74 & 31.0 \\
Low Social Support & 162 & 100 \\
\hline Medium Social Support & 106 & \\
High Social Support & 342 & \\
Total & & \\
\hline
\end{tabular}

Table (6) Distribution the scores of EORTC QoL C-30.

\begin{tabular}{lcccc}
\hline EORTC QoL C-30 Subscales & Mean & SD & Minimum & Maximum \\
Global Health Status/QoL & 68.52 & 19.80 & 16.67 & $\mathbf{1 0 0}$ \\
EORTC QoL C-30 Functions & & & & \\
Physical Function & 66.12 & 20.11 & 13.33 & $\mathbf{1 0 0}$ \\
Role Functioning & 68.42 & 25.87 & 0.00 & $\mathbf{1 0 0}$ \\
Emotional Function & 52.10 & 29.60 & 0.00 & $\mathbf{1 0 0}$ \\
Cognitive Function & 54.97 & 26.99 & 0.00 & $\mathbf{1 0 0}$ \\
Social Function & 67.54 & 28.31 & 0.00 & $\mathbf{1 0 0}$ \\
Total QoL C-30 Functions & 61.83 & 17.61 & 22.67 & $\mathbf{9 8 . 6 7}$ \\
EORTC QoL C-30 Symptoms & & & & \\
Fatigue & 43.60 & 21.16 & 0.00 & $\mathbf{8 8 . 8 9}$ \\
Nausea & 7.60 & 14.03 & 0.00 & $\mathbf{6 6 . 6 7}$ \\
Pain & 39.96 & 24.15 & 0.00 & $\mathbf{6 6 . 6 7}$ \\
Dyspnea & 16.37 & 18.21 & 0.00 & $\mathbf{1 0 0}$ \\
Insomnia & 29.24 & 27.82 & 0.00 & $\mathbf{6 6 . 6 7}$ \\
\hline Appetite Loss & 13.65 & 22.48 & 0.00 & $\mathbf{6 6 . 6 7}$ \\
Constipation & 18.23 & 27.81 & 0.00 & $\mathbf{1 0 0}$ \\
Diarrhea & 3.70 & 11.11 & 0.00 & $\mathbf{6 6 . 6 7}$ \\
Financial Deficit (Difficulties) & 55.95 & 33.07 & 0.00 & $\mathbf{1 0 0}$ \\
\hline Total QoL C-30 Symptoms & 25.37 & 14.08 & 0.00 & $\mathbf{5 7 . 4 1}$ \\
\hline
\end{tabular}

Table (7) Distribution the scores of EORTC QoL BR-23.

\begin{tabular}{lllll}
\hline EORTC QoL BR-23 Subscales & Mean & SD & Minimum & Maximum \\
BR-23 Functions & & & & \\
Body Image & 52.88 & 19.97 & 8.34 & 100 \\
\hline
\end{tabular}




\begin{tabular}{|lllll}
\hline Future Perspective & 58.87 & 29.44 & 0.00 & $\mathbf{1 0 0}$ \\
Sex Function & 32.99 & 28.79 & 0.00 & $\mathbf{1 0 0}$ \\
Sexual Enjoyment & 45.66 & 38.88 & 0.00 & $\mathbf{1 0 0}$ \\
\hline Total BR-23 Functions & 45.81 & 17.83 & 2.08 & $\mathbf{9 7 . 9 2}$ \\
BR-23 Symptoms & & & & $\mathbf{7 6 . 1 9}$ \\
Systematic Therapy Side effect & 41.35 & 18.53 & 0.00 & $\mathbf{1 0 0}$ \\
Hair Loss & 59.84 & 41.37 & 0.00 & $\mathbf{8 8 . 8 9}$ \\
\hline Arm Symptom & 43.27 & 25.05 & 0.00 & $\mathbf{7 5 . 0 0}$ \\
\hline Breast Symptom & 32.36 & 22.27 & 0.00 & $\mathbf{8 2 . 9 4}$ \\
\hline Total BR 23 Symptoms & 44.21 & 19.76 & 0.00 & \\
\hline
\end{tabular}

Table (8) Distribution of correlation among study dependent variables.

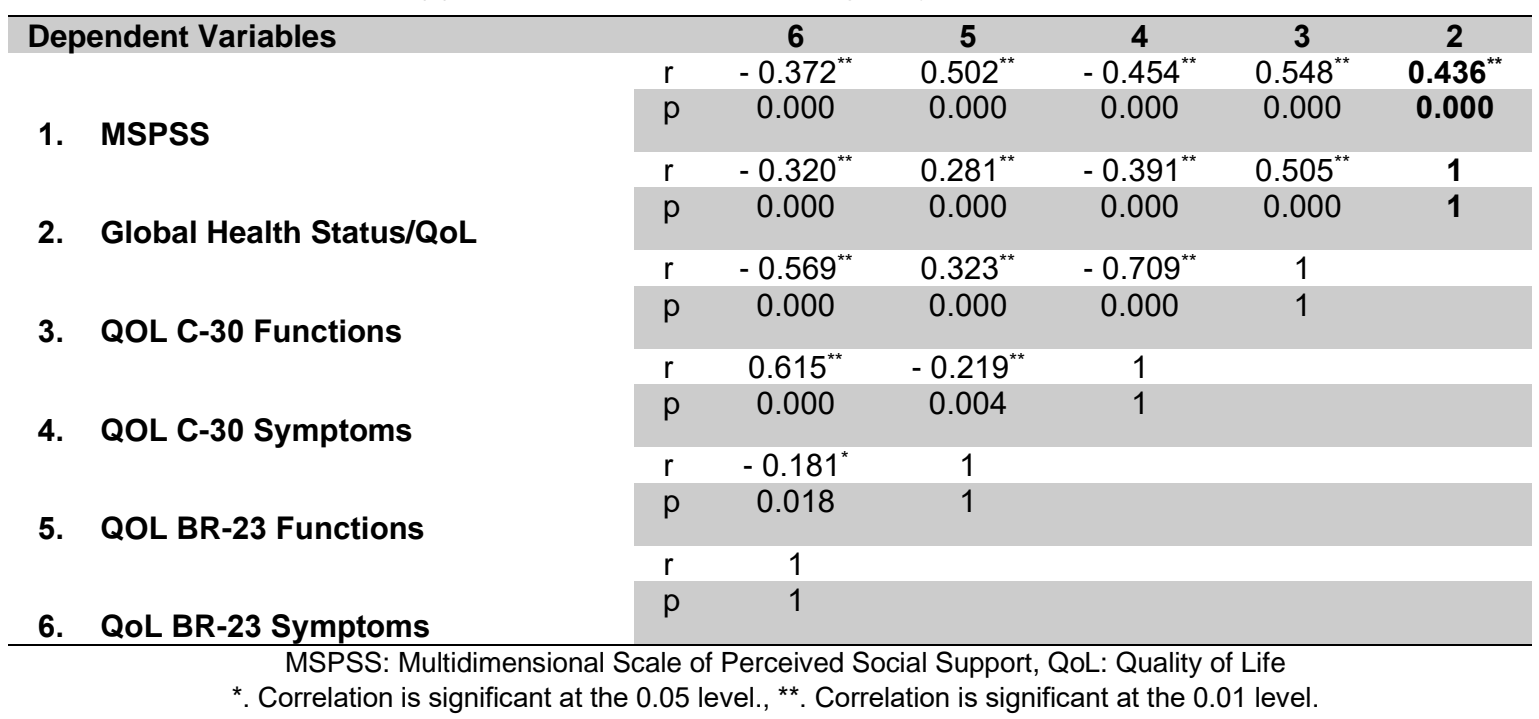

Table (9) The difference of QOL-C30 scores according to levels of social support.

\begin{tabular}{|c|c|c|c|c|c|}
\hline \multirow[t]{2}{*}{ QOL-C30 Subscales } & \multicolumn{3}{|c|}{ Levels of Social Support } & \multirow[t]{2}{*}{$\mathbf{F}$} & \multirow[t]{2}{*}{$\mathbf{p}$} \\
\hline & Low & Moderate & High & & \\
\hline Global Health/QoL & $57.66 \pm 24.95$ & $67.39 \pm 16.94$ & $77.83 \pm 15.32$ & 13.22 & 0.000 \\
\hline \multicolumn{6}{|l|}{ C-30 Functional Subscales } \\
\hline Physical Function & $56.40 \pm 21.06$ & $63.95 \pm 19.29$ & $76.23 \pm 16.25$ & 13.13 & 0.000 \\
\hline Role Function & $58.49 \pm 16.55$ & $67.57 \pm 28.85$ & $75.31 \pm 27.53$ & 7.29 & 0.001 \\
\hline Emotional & $31.53 \pm 24.54$ & $50.62 \pm 31.84$ & $68.71 \pm 17.37$ & 21.60 & 0.000 \\
\hline Cognitive & $42.34 \pm 23.44$ & $50.82 \pm 28.74$ & $70.13 \pm 18.88$ & 15.67 & 0.000 \\
\hline Social & $46.40 \pm 20.09$ & $67.08 \pm 27.89$ & $83.02 \pm 17.75$ & 22.97 & 0.000 \\
\hline Total C-30 Functions & $48.85 \pm 15.08$ & $61.56 \pm 18.67$ & $71.31 \pm 10.42$ & 21.18 & 0.000 \\
\hline \multicolumn{6}{|l|}{ C-30 Symptoms Subscales } \\
\hline Fatigue & $52.85 \pm 20.52$ & $47.87 \pm 22.54$ & $30.61 \pm 23.96$ & 13.33 & 0.000 \\
\hline Nausea & $11.26 \pm 18.86$ & $7.20 \pm 12.62$ & $5.66 \pm 11.76$ & 1.82 & 0.166 \\
\hline Pain & $52.25 \pm 17.20$ & $42.59 \pm 23.72$ & $27.36 \pm 23.59$ & 14.47 & 0.000 \\
\hline Dyspnea & $24.32 \pm 16.94$ & $16.87 \pm 18.35$ & $10.06 \pm 16.77$ & 7.23 & 0.001 \\
\hline
\end{tabular}




\begin{tabular}{|c|c|c|c|c|c|}
\hline Insomnia & $46.85 \pm 20.41$ & $29.22 \pm 26.03$ & $16.98 \pm 25.84$ & 14.56 & 0.000 \\
\hline Appetite Loss & $24.32 \pm 20.40$ & $9.05 \pm 19.01$ & $13.21 \pm 23.88$ & 6.23 & 0.002 \\
\hline Constipation & $17.12 \pm 25.61$ & $21.40 \pm 29.96$ & $14.46 \pm 25.75$ & 1.04 & 0.355 \\
\hline Diarrhea & $3.60 \pm 10.49$ & $4.12 \pm 12.23$ & $3.15 \pm 9.84$ & 0.12 & 0.884 \\
\hline Financial Deficit & $68.47 \pm 28.27$ & $58.03 \pm 32.39$ & $44.03 \pm 33.83$ & 6.67 & 0.002 \\
\hline Total C-30 Symptoms & $33.45 \pm 11.25$ & $26.26 \pm 12.36$ & $18.39 \pm 15.10$ & 14.58 & 0.000 \\
\hline
\end{tabular}

Table (10) The difference of QOL-BR23 scores according to levels of social support.

QOL-BR-23 Subscales

\begin{tabular}{|c|c|c|c|c|c|}
\hline \multirow[t]{2}{*}{ QOL-BR-23 Subscales } & \multicolumn{3}{|c|}{ Levels of Social Support } & \multirow[t]{2}{*}{$\mathbf{F}$} & \multirow[t]{2}{*}{ p } \\
\hline & Low & Medium & High & & \\
\hline \multicolumn{6}{|l|}{ BR-23 Functional Subscales } \\
\hline Body Image & $47.52 \pm 20.21$ & $51.44 \pm 22.47$ & $58.81 \pm 13.62$ & 4.01 & 0.020 \\
\hline Future Perspective & $45.05 \pm 22.52$ & $59.26 \pm 32.49$ & $67.93 \pm 25.29$ & 7.061 & 0.001 \\
\hline Sex Function & $17.33 \pm 26.56$ & $33.12 \pm 28.79$ & $40.97 \pm 27.06$ & 5.914 & 0.003 \\
\hline Sexual Enjoyment & $50.41 \pm 48.57$ & $44.29 \pm 37.76$ & $44.44 \pm 33.10$ & 0.35 & 0.705 \\
\hline Total BR-23 Functions & $36.37 \pm 15.60$ & $45.83 \pm 19.02$ & $52.36 \pm 14.39$ & 9.65 & 0.000 \\
\hline \multicolumn{6}{|l|}{ BR-23 Symptoms Subscales } \\
\hline $\begin{array}{l}\text { Systematic Therapy Side } \\
\text { Effect }\end{array}$ & $45.18 \pm 18.69$ & $44.12 \pm 17.37$ & $33.83 \pm 17.05$ & 6.905 & 0.001 \\
\hline Hair Loss & $62.59 \pm 41.29$ & $62.33 \pm 40.84$ & $50.49 \pm 42.03$ & 1.221 & 0.297 \\
\hline Arm Symptoms & $54.95 \pm 18.32$ & $44.22 \pm 16.83$ & $33.79 \pm 22.08$ & 8.610 & 0.000 \\
\hline Breast Symptoms & $41.14 \pm 22.08$ & $35.25 \pm 22.60$ & $22.14 \pm 18.00$ & 10.139 & 0.000 \\
\hline Total BR-23 Symptoms & $50.97 \pm 18.83$ & $46.73 \pm 17.98$ & $35.06 \pm 19.57$ & 4.01 & 0.020 \\
\hline
\end{tabular}

\section{DISCUSSION}

This study assessed the associations between social support and QOL in a sample of Kurdish breast cancer women. Present study participants' QOL seemed to be affected by breast cancer and, particularly, the emotion, cognitive, sexual function, and sexual enjoyment functions of QOL, on another hand financial deficit, fatigue, pain, hair loss, therapy adverse effect, and arm symptom were a most disrupting symptom. According to the literature, hair loss is one of the most important side effects of chemotherapy (Yfantis et al 2020). Monfared et al. (2013) in their study, found that QoL was lower in the emotional area compared to other areas.

Regarding the QLQ-BR23 questionnaire, in Yfantis et al (2020) study, all investigated symptoms were at fairly favorable levels in their study. The highest-scoring (worse) symptoms/items were fatigue, followed by insomnia and financial problems. Our results strengthen findings from previous studies that report significant problems including fatigue, pain in the joints, and sleeping disturbances (Schmidt et al, 2019). Economic difficulties are negatively correlated with QoL and as the functional status of breast cancer, women impair, more adverse economic situations appear, the financial deficit remains a major issue in breast cancer care. A significant number of cancer patients and families struggle with financial difficulty (2020).

As expected, the family was the greatest source of social support for participants, followed by significant others such as nurses and physicians, and friend was the last source of support stated by the patients. In eastern societies, particularly in Kurdish cultural family is an umbrella for family members, there is a strong relationship among members, taking care of sick members or during illness is the principle and necessity. 
The finding of present study indicated that the breast cancer patients in who perceived higher social support showed better global health status / QoL, social, physical, role functioning, cognitive, and emotional functions, and reported lower fatigue, pain, dyspnea, insomnia, appetite loss, and financial deficit symptoms significantly, $(p<0.05)$, Table (8). Furthermore, breast cancerrelated functions such as body image, future perspective, and sex function was significantly higher among patients perceived higher social support. Systematic breast cancer therapy side effect, arm and breast symptoms were lower among higher social support group.

Social support has been shown to be significantly important in helping people manage life crises, health challenges, and improving QoL. The sources of support in the present study were family, significant others (health care provider), and friends, with family being the most provider. Similarly, a study conducted in Ghana found that breast cancer patients received support from their families, spouses, friends, health professionals, and spiritual leaders (Benso et al 2020). In addition, Salakari et al (2017) considered the spouse's or the partner's as main provider of social support during the disease and recovery phase of breast cancer. Even though the need of social support is increased in the recovery phase. Spouses, children, families, and friends were dominant in providing forms of support to these women. This support findings from previous study, which indicated that spouses and families were supportive, giving confidence, listening to the patients, and supporting them in the decisionmaking process about the treatment (Adamand and Koranteng 2020). Furthermore, Adamand and Koranteng (2020) found that most women diagnosed with $\mathrm{BC}$ receive helpful informational and emotional support from healthcare providers. They reported that these supports are important for the psychological well-being of the patients especially in the early stages of diagnosis, where patients face the greatest anxiety and the absence of it could hamper effective healthcare access by breast cancer patients. Support from friends was less in this study compared with other sources of support.

Yang et al (2017) reported that the QoL of breast cancer patients in preoperative, postoperative chemoradiotherapy and rehabilitation periods were all positively correlated with the overall social support (all $\mathrm{P}<0.01)$, the researchers concluded that the QoL of breast cancer patients at different periods of treatment is positively correlated with the social support. The quality of life can be enhanced by improving the social support for the patients. In addition, Zhang et al (2020) study has added to the evidence that the positive resources of hope and social support were positively associated with QoL in oral cancer. Furthermore, a German (Zenger et al., 2013) and Colombian (Finck et al 2018) studies found that cancer patients want and indeed get social support from their physicians, nurse and families/friends, while other professionals (psychologists, social workers, pastors) are less involved.

The finding of this study provides an understanding of the importance of social support, especially from family and significant others, for women who underwent breast cancer treatment. As reported by Spatuzzi et al (2016), interventions that involve family and partners should be aimed at improving communication and facilitating both the patients' and the family members' expressions of needs and feelings.

A recent study highly indicated social support as an important factor in improving QOL, recommended that positive social support would be beneficial to improve the QOL of breast cancer patients, and providing targeted support for the breast cancer patients, such as positive interventions of expressive support, will be helpful to improve their QOL as well as relieve their fear of disease in the oncology field (Ban et al 2021).

\section{CONCLUSIONS}

The results present study indicated that family as a greatest source of social support, women with breast cancer had a satisfactory level of global health status/QoL, role, social, and physical functioning, as they scored high in functionality sub-scales. Emotional function was more affected area. Regarding the symptoms' subscale, financial deficit, fatigue, and pain, were the most important problems. Similarly, patients scored better in functional scale (QLQ-BR-23), future perspective and body image. Hair loss, arm symptoms, and systemic therapy side effects were the most disturbing symptoms. Patients with high social support showed better QoL. Some issues, for instance, financial deficit, hair loss, fatigue, insomnia, etc., warrant good supportive therapy to reduce the concerns of patients and to give them psychological support, health care professionals can play a role in reducing burdens through informational and emotional support. Future studies can be conduct keeping in view specific problems in detail and roles of social support in enhancing QoL.

\section{ETHICAL CONSIDERATIONS COMPLIANCE WITH ETHICAL GUIDELINES}

An administrative agreement was obtained from head of the clinical nursing department, and approved by the College of Nursing, University of Sulaimani, Iraq. The participants were informed about the research's purpose and ensured anonymity and confidentiality of the information. A written informed, voluntary participation consent was obtained from each participant.

\section{FUNDING}

This research did not receive any grant from funding agencies in the public, commercial, or non-profit sectors. 


\section{AUTHOR'S CONTRIBUTIONS}

Study concept; Writing the original draft; Data collection; Data analysis and Reviewing the final edition by all authors.

DISCLOSURE STATEMENT: The authors report no conflict of interest.

\section{ACKNOWLEDGEMENTS}

We thank the anonymous referees for their useful suggestions.

\section{REFERENCES}

Adam A, and Koranteng F. (2020) Availability, accessibility, and impact of social support on breast cancer treatment among breast cancer patients in Kumasi, Ghana: A qualitative study. PLoS ONE 15(4): e0231691. https://doi.org/10.1371/journal.pone.0231691.

Adam A, and Koranteng F. (2020). Availability, accessibility, and impact of social support on breast cancer treatment among breast cancer patients in Kumasi, Ghana: A qualitative study. PLoS ONE 15(4): e0231691. https://doi.org/10.1371/journal.pone.0231691. Alshraifeen, A., Sami Al-Rawashdeh, K., Alzoubi, F., Tanash, M., Al-Hawamdih, A., and AlGhabeesh, S., (2020) Social support predicted quality of life in people receiving haemodialysis treatment: A cross-sectional survey, 7(5); pp: 1517-1525. https://doi.org/10.1002/nop2.533. Ban, Y., Li, M., Yu, M. (2021) The effect of fear of progression on quality of life among breast cancer patients: the mediating role of social support. Health Qual Life Outcomes 19, 178 (2021). https://doi.org/10.1186/s12955-021-01816-7.

Canty-Mitchell J, Zimet GD. (2000) Psychometric properties of the Multidimensional Scale of Perceived Social Support in urban adolescents. Am J Community Psychol. 28(3):391-400. doi: 10.1023/A:1005109522457. PMID: 10945123.

Celik GK, Cakir H, Kut E. (2021). Mediating role of social support in resilience and quality of life in patients with breast cancer: structural equation model analysis. Asia Pac J Oncol Nurs. 8(1):86-93. Coughlin, S. S., Ayyala, D. N., Tingen, M. S., \& Cortes, J. E. (2020). Financial distress among breast cancer survivors. Current cancer reports, 2(1), 48-53. https://doi.org/10.25082/CCR.2020.01.004 Fayers PM, Aaronson NK, Bjordal K, Groenvold M, Curran D, Bottomley A, (2001) On behalf of the EORTC Quality of Life Group. EORTC QLQ-C30 scoring manual. 3rd ed. Brussels: European
Organization for Research and Treatment of Cancer; 2001. p. 2001.

Finck, C., Barradas, S., Zenger, M., \& Hinz, A. (2018). Quality of life in breast cancer patients: Associations with optimism and social support. International journal of clinical and health psychology : IJCHP, 18(1), 27-34. https://doi.org/10.1016/j.ijchp.2017.11.002.

Hassen, A. M., Taye, G., Gizaw, M., \& Hussien, F. M. (2019). Quality of life and associated factors among patients with breast cancer under chemotherapy at Tikur Anbessa specialized hospital, Addis Ababa, Ethiopia. PloS one, 14(9), e0222629.

https://doi.org/10.1371/journal.pone.0222629. Kim J, Han JY, Shaw B, McTavish F, Gustafson D. (2010). The roles of social support and coping strategies in predicting breast cancer patients' emotional well-being: testing mediation and moderation models. Journal of health psychology., 15(4):543-52. pmid:20460411.

Monfared A, Pakseresht S, Ghanbari Khanghah A, Atrkar-Roshan Z. (2013) Health-related quality of life and its related factors among women with breast cancer. J Holist Nurs Midwifery. 23(2):5262.

Oh PJ, Cho JR. (2020). Changes in fatigue, psychological distress, and quality of life after chemotherapy in women with breast cancer: a prospective study. Cancer Nurs., 43(1): E54-60.

Salakari M, Pylkkanen L, Sillanmaki L, Nurminen R, Rautava P, Koskenvuo M, and Suominen S (2017) Social support and breast cancer: A comparatory study of breast cancer survivors, women with mental depression, women with hypertension and healthy female controls,; The Breast 35, pp: 85-90. .doi.org/10.1016/j.breast.2017.06.017.

Schmidt ME, Scherer S, Wiskemann J, Steindorf K. Return to work after breast cancer: the role of treatment-related side effects and potential impact on quality of life. Eur J Cancer Care. 2019;28(4):e13051.

Siegel RL, Miller KD, Jemal A. Cancer statistics, (2017). CA Cancer J Clin. 2017;67(1):7-30. 10.3322/caac. 21387.

Spatuzzi R, Vespa A, Lorenzi P, Miccinesi G, Ricciuti M, Cifarelli W, Susi M, Fabrizio T, Ferrari M, G, Ottaviani M, Giulietti M, V, Merico F, Aieta M. (2016) Evaluation of Social Support, Quality of Life, and Body Image in Women with Breast Cancer. Breast Care, 11, pp: 28-32. doi: 10.1159/000443493.

Sprangers MA, Groenvold M, Arraras JI, Franklin J, Velde A. (1996) The European Organization for Research and Treatment of Cancer breast cancerspecific quality-of-life questionnaire module: first 
results from a three-country field study. J Clin Oncol., 14:2756-2768.

Wang YH, Li JQ, Shi JF, Que JY, Liu JJ, Lappin JM. (2020) Depression and anxiety in relation to cancer incidence and mortality: a systematic review and meta-analysis of cohort studies. Mol Psychiatry. 2020;25(7):1487-99.

Yang L, Song WP, Chen ZL, Wang Y, Chen YY, Hua YH, Chen M, Zou WB. (2017) Correlation between social support and quality of life in patients with breast cancer at different periods of treatment. Zhonghua Zhong Liu Za Zhi., 3;39(3):202-206.

Chinese. doi: $10.3760 / \mathrm{cma}$.j.issn.02533766.2017.03.009. PMID: 28316220.

Yfantis, A., Sarafis, P., Moisoglou, I., Tolia, M., Intas, G., Tiniakou, I., Zografos, K., Zografos, G., Constantinou, M., Nikolentzos, A. \& Kontos, M . (2020) How breast cancer treatments affect the quality of life of women with non-metastatic breast cancer one year after surgical treatment: a cross-sectional study in Greece. BMC Surg 20, p 210 . https://doi.org/10.1186/s12893-020-00871$z$.

Zenger M., Finck C., Zanon C., Jimenez W., Singer S., Hinz A. (2013) Evaluation of the Latin American version of the Life Orientation Test-Revised. International Journal of Clinical and Health Psychology. 13, pp: 243-252.

Zhang, Y., Cui, C., Wang. (2020) Effects of stigma, hope and social support on quality of life among Chinese patients diagnosed with oral cancer: a cross-sectional study. Health Qual Life Outcomes 18, 112. https://doi.org/10.1186/s12955-02001353-9.

Zimet GD, Dahlem NW, Zimet SG, Farley GK. (1988) The Multidimensional Scale of Perceived Social Support. Journal of Personality Assessment 52:30-41. 